\title{
Simple model of a multi-batch driven pipeline
}

\author{
Sašo Blažič ${ }^{a}, *$ Drago Matko ${ }^{a}$, Gerhard Geiger ${ }^{b}$ \\ ${ }^{\text {a }}$ Faculty of Electrical Engineering, University of Ljubljana, Ljubljana, Slovenia \\ ${ }^{\mathrm{b}}$ Fachhochschule Gelsenkirchen, Germany
}

Received 28 January 2003; received in revised form 18 November 2003; accepted 19 November 2003

\begin{abstract}
The problem of modelling and simulating pipelines that are used for transporting different fluids is addressed in the paper. The problem is solved by including fluid density in the model beside pressure and velocity of the medium. First, the system of nonlinear partial differential equations is derived. Then, the obtained model is linearised and transformed into the transfer function form with three inputs and three outputs. Four different forms of model description are presented in the paper. Since transfer functions are transcendent, they cannot be simulated using classical tools. Rational transfer function approximation of the model was found and that simple model was validated on the real industrial pipeline. It was also compared to the model that does not take the changes in fluid density into account. The latter model cannot cope with batch changes whereas the proposed one can.
\end{abstract}

(C) 2003 IMACS. Published by Elsevier B.V. All rights reserved.

Keywords: Pipeline; Simulation; Transcendent transfer function; Lumped parameter model

\section{Introduction}

Real time transient model (RTTM) based leak monitoring systems require a sophisticated mathematical model of the flow in pipelines. The so called "water hammer equations" are relatively simple mathematical models assuming isentropic flow; they are obtained using the principles of mass and momentum conservation [1].

However, in the case when different fluids are transported through the same pipeline, the above model is not adequate. The water hammer equation can easily be extended, as will be shown in Section 2 . This enables simplified description of multi-product-flows with multiple products or batches being transported at the same time in one pipeline. Up to now, there is no analytical solution for this nonlinear, partial differential equation system available. Instead, numerical solution techniques like the method of characteristics can be used [2].

* Corresponding author. Tel.: +386-1-4768-763; fax: +386-1-4264-631.

E-mail address: saso.blazic@fe.uni-lj.si (S. Blažič). 
Another possibility to solve the problem is to use linearisation and Laplace transformation techniques in order to get a frequency domain description. This leads to a simplified pipeline model with lumped parameters. We hereby get some advantages: the classical system theory for multi-input multi-output (MIMO) systems can be used, e.g. for controller design and system identification. The resulting algorithms are less time-consuming and hence better suited for critical real time applications. Additionally, the analysis of fluid transients caused by leaks is much easier.

In Section 2, the nonlinear model of a pipeline is derived that takes into account multiple fluids being transported. In Section 3, the model is linearised, and in Section 4, a simplified model with lumped parameters is given. The obtained models is compared to the one derived in [3] that assumes constant density of the fluid. The results are validated on the real industrial pipeline in Section 5. At the end some conclusions are given.

\section{Mathematical model of the pipeline}

The classical solution for unsteady flow problems is obtained by using the equations for continuity, momentum, and energy. These equations correspond to the physical principles of mass, momentum, and energy conservation. Applying these equations leads to a coupled nonlinear set of partial differential equations and hence, they are very difficult to solve analytically. To date, there is no general closed-form solution. Further problems arise in the case of turbulent flow, which introduces stochastic flow behaviour. Therefore, the mathematical derivation for the flow through a pipeline is a mixture of both theoretical and empirical approaches.

The following assumptions for the derivation of a mathematical model of the flow through pipelines are made:

1. Fluid is compressible. Compressibility of fluid results in an unsteady flow.

2. Flow is viscous. Viscosity causes shear stresses in a moving fluid.

3. Flow is adiabatic. No transfer of energy between fluid and pipeline will be considered.

4. Flow is isothermal. Temperature changes due to pressure changes can be neglected for liquids. Under these circumstances, temperature changes could only be the result of friction effects, but these effects will also be neglected. Therefore, the temperature along the pipeline is constant.

5. Flow is one-dimensional. All characteristics of the pipeline such as velocity $v$ and pressure $p$ depend only on the $x$-axis laid along the pipeline.

Consider now a pipeline of length $L_{\mathrm{p}}$ with constant diameter

$$
D=D(x)=2 R=\text { constant }
$$

The continuity equation in conservative form for the one-dimensional case yields [4]

$$
\frac{\mathrm{d} \rho}{\mathrm{d} t}+\rho \frac{\partial v}{\partial x}=0
$$

with density $\rho(x)$, velocity $v(x)$, and with the substantial or total derivative

$$
\frac{\mathrm{d} \rho}{\mathrm{d} t} \equiv \frac{\partial \rho}{\partial t}+v \frac{\partial \rho}{\partial x}
$$


The momentum equation in conservative form for the one-dimensional case yields [4]

$$
\rho \frac{\mathrm{d} v}{\mathrm{~d} t}=-\rho g \sin \alpha-\frac{\partial p}{\partial x}+\frac{\partial p_{\mathrm{L}}}{\partial x}
$$

with pressure $p(x)$. The quantity $g \sin \alpha$ is the $x$-component of the standard gravity vector $g$. The pressure loss $p_{\mathrm{L}}$ rely on the shear stress $\tau_{\mathrm{R}}$. The formula from Darcy and Weisbach [5] states that

$$
\frac{\partial p_{\mathrm{L}}}{\partial x}=-\rho \frac{\lambda v|v|}{2 D}
$$

with the dimensionless friction coefficient $\lambda(v)$. This equation holds for laminar flow as well as for turbulent flow. Laminar flow is described by [5]

$$
\lambda=\lambda(v)=\frac{64}{R e}
$$

if the dimensionless Reynolds number

$$
R e=\frac{D}{v} v
$$

is smaller than 2320 ( $v$ is the kinematic viscosity of the fluid). For larger values of the Reynolds number, flow is assumed to be turbulent. In that case, Eq. (6) can be replaced by an appropriately mixed theoretically and empirically derived formula such as the formula of Colebrook [5]

$$
\frac{1}{\sqrt{\lambda}}=-2 \log \left(\frac{2.51}{\operatorname{Re} \sqrt{\lambda}}+0.27 \frac{k_{\mathrm{R}}}{D}\right)
$$

with roughness height $k_{\mathrm{R}}$ as a measure of the roughness of commercial pipes.

Using Eqs. (4) and (5) we obtain

$$
\frac{\mathrm{d} v}{\mathrm{~d} t}+\frac{1}{\rho} \frac{\partial p}{\partial x}+g \sin \alpha+\rho \frac{\lambda v|v|}{2 D}=0
$$

The model of the pipeline is completed by

$$
p=a^{2} \rho
$$

with the (isentropic) speed of sound $a$ of the fluid. Eqs. (2), (9) and (10) lead to the following mathematical model that will be treated in the paper:

$$
\begin{aligned}
& \frac{\mathrm{d} \rho}{\mathrm{d} t}+\rho \frac{\partial v}{\partial x}=0 \\
& \frac{\mathrm{d} v}{\mathrm{~d} t}+\frac{1}{\rho} \frac{\partial p}{\partial x}+g \sin \alpha+\frac{\lambda v|v|}{2 D}=0 \\
& \frac{\mathrm{d} p}{\mathrm{~d} t}-a^{2} \frac{\mathrm{d} \rho}{\mathrm{d} t}=0
\end{aligned}
$$




\section{Linearisation of the mathematical model}

By replacing total derivatives with partial ones, multiplying Eq. (12) with $\rho$, and by inserting Eq. (11) into Eq. (13) we get

$$
\begin{aligned}
& \frac{\partial \rho}{\partial t}+v \frac{\partial \rho}{\partial x}+\rho \frac{\partial v}{\partial x}=0 \\
& \rho \frac{\partial v}{\partial t}+\rho v \frac{\partial v}{\partial x}+\frac{\partial p}{\partial x}+\rho g \sin \alpha+\frac{\rho \lambda v|v|}{2 D}=0 \\
& \frac{\partial p}{\partial t}+v \frac{\partial p}{\partial x}+a^{2} \rho \frac{\partial v}{\partial x}=0
\end{aligned}
$$

In order to simplify this system of partial differential equations, first the steady state will be evaluated. It is obtained by setting to zero all partial derivatives with respect to time $(\partial / \partial t:=0)$ which yields the following set of ordinary differential equations (with respect to $x$ ):

$$
\begin{aligned}
& \bar{v} \frac{\mathrm{d} \bar{\rho}}{\mathrm{d} x}+\bar{\rho} \frac{\mathrm{d} \bar{v}}{\mathrm{~d} x}=0 \\
& \bar{\rho} \bar{v} \frac{\mathrm{d} \bar{v}}{\mathrm{~d} x}+\frac{\mathrm{d} \bar{p}}{\mathrm{~d} x}+\bar{\rho} g \sin \alpha+\frac{\bar{\rho} \lambda \bar{v}|\bar{v}|}{2 D}=0 \\
& \bar{v} \frac{\mathrm{d} \bar{p}}{\mathrm{~d} x}+a^{2} \bar{\rho} \frac{\mathrm{d} \bar{v}}{\mathrm{~d} x}=0
\end{aligned}
$$

This set of equations cannot be solved analytically. It was solved by MATLAB-SIMULINK for the pipeline used for the verification of the model (see Section 5). The results for $\bar{p}, \bar{v}$ and $\bar{\rho}$ are shown in Fig. 1.

The convective terms $\rho v \partial v / \partial x$ and $v \partial p / \partial x$ in Eqs. (15) and (16), respectively, are small compared to the derivatives with respect to time $\rho \partial v / \partial t$ and $\partial p / \partial t$, respectively, and will be neglected. Next, Eqs. (15) and (16) will be linearised around steady-state solution. In order to do this, new variables will be introduced:

$$
\begin{aligned}
& \tilde{v}(x, t)=v(x, t)-\bar{v}(x) \\
& \tilde{p}(x, t)=p(x, t)-\bar{p}(x) \\
& \tilde{\rho}(x, t)=\rho(x, t)-\bar{\rho}(x)
\end{aligned}
$$

After linearising Eqs. (15) and (16), and taking into account Eqs. (18) and (19), the following is obtained:

$$
\begin{aligned}
& \bar{\rho} \frac{\partial \tilde{v}}{\partial t}+\frac{\partial \tilde{p}}{\partial x}+\tilde{\rho} g \sin \alpha+\frac{\bar{\rho} \lambda|\bar{v}|}{D} \tilde{v}+\frac{\lambda \bar{v}|\bar{v}|}{2 D} \tilde{\rho}=0 \\
& \frac{\partial \tilde{p}}{\partial t}+a^{2} \bar{\rho} \frac{\partial \tilde{v}}{\partial x}+a^{2} \tilde{\rho} \frac{\mathrm{d} \bar{v}}{\mathrm{~d} x}=0
\end{aligned}
$$

The last term in Eq. (24) can be neglected due to the fact that $\mathrm{d} \bar{v} / \mathrm{d} x$ is very small (see Fig. 1). Using notations that are common in the analysis of electrical transmission lines:

$$
L=\bar{\rho}
$$



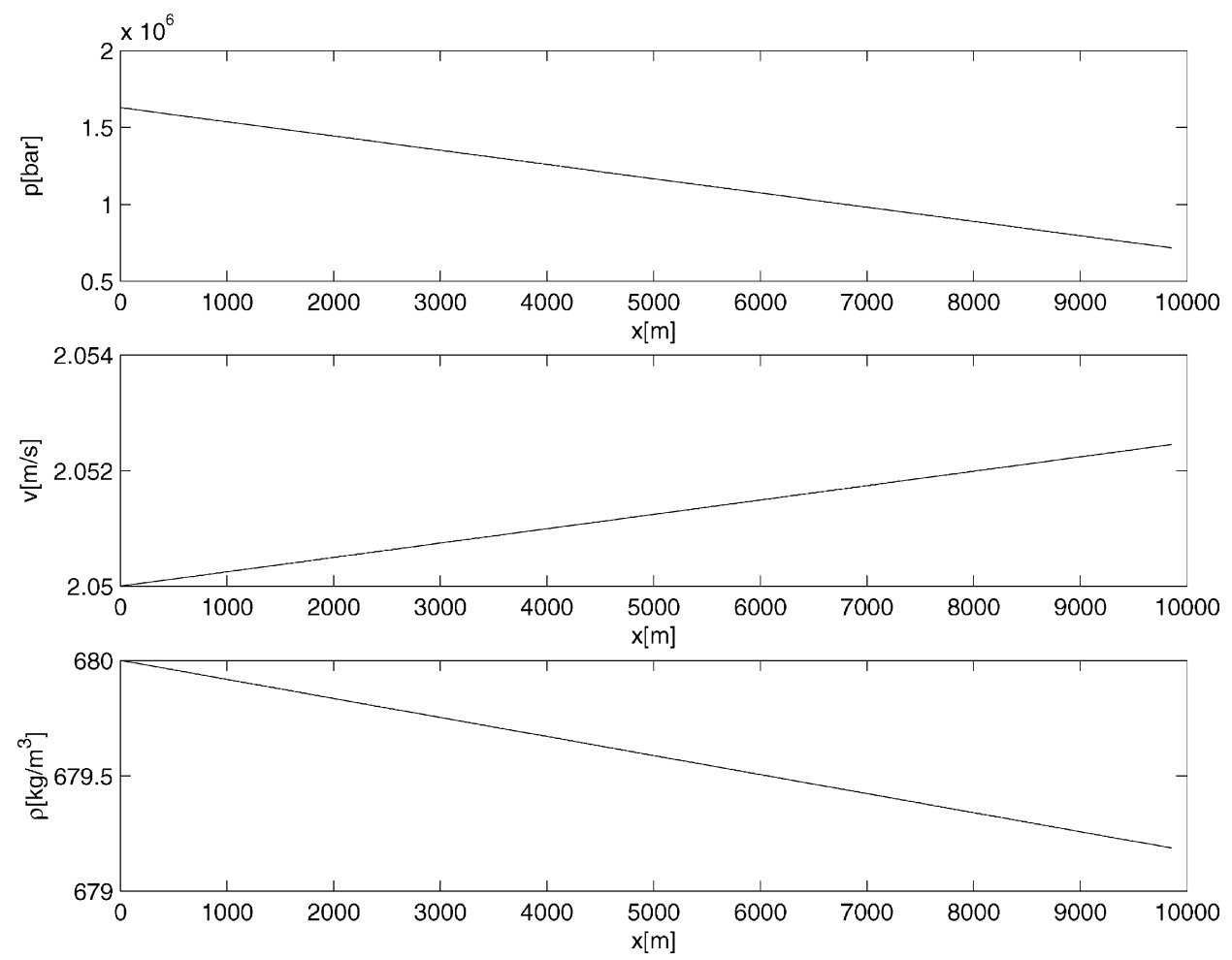

Fig. 1. Steady state solution of the pipeline: pressure $\bar{p}$, velocity $\bar{v}$, and density $\bar{\rho}$.

$$
\begin{aligned}
R & =\frac{\bar{\rho} \lambda|\bar{v}|}{D} \\
C & =\frac{1}{a^{2} \bar{\rho}}
\end{aligned}
$$

and by denoting

$$
T=g \sin \alpha+\frac{\lambda \bar{v}|\bar{v}|}{2 D}
$$

we get

$$
\begin{aligned}
& L \frac{\partial \tilde{v}}{\partial t}+R \tilde{v}+T \tilde{\rho}=-\frac{\partial \tilde{p}}{\partial x} \\
& C \frac{\partial \tilde{p}}{\partial t}=-\frac{\partial \tilde{v}}{\partial x}
\end{aligned}
$$

Since the fluid in the pipeline is almost incompressible, the dynamics of $\rho$ in Eq. (14) are relatively slow, compared to those of $v$ and $p$ in Eqs. (15) and (16). As a consequence, the velocity profile along the pipeline is nearly constant within each time instant. Due to a special reason that will be explained later, 
the derivative $\partial v / \partial x$ will not be neglected in Eq. (14). Rather, it will be approximated with a very small constant $\Delta v_{x}$. Linearising (14) and considering (22) then yields:

$$
\frac{\partial \tilde{\rho}(x, t)}{\partial t}+\tilde{v}(x, t) \frac{\mathrm{d} \bar{\rho}(x)}{\mathrm{d} x}+\bar{v}(x) \frac{\partial \tilde{\rho}(x, t)}{\partial x}+\tilde{\rho}(x, t) \Delta v_{x}=0
$$

Note that $\mathrm{d} \bar{\rho} / \mathrm{d} x$ is very small (see Fig. 1). It could be neglected in Eq. (31), but it will not be at this point since its influence is similar to the influence of $\mathrm{d} \bar{v} / \mathrm{d} x$ (see Eq. (17)).

The first step in obtaining analytical solution of the system is to solve Eq. (31) by applying Laplace transformation on it. It is assumed that it is permissible to interchange the order of differentiation with respect to $x$ and the taking of the Laplace transform. The consequence is that the first-order ordinary differential equation is obtained [6]

$$
s \tilde{\rho}(x, s)-\tilde{\rho}(x, 0)+\tilde{v}(x, s) \frac{\mathrm{d} \bar{\rho}(x)}{\mathrm{d} x}+\bar{v}(x) \frac{\mathrm{d} \tilde{\rho}(x, s)}{\mathrm{d} x}+\Delta v_{x} \tilde{\rho}(x, s)=0
$$

It is assumed here that the system rests at time $t=0$, i.e. $\tilde{\rho}(x, 0)=0, \tilde{p}(x, 0)=0, \tilde{v}(x, 0)=0$. Eq. (32) can then be transformed to

$$
\frac{\mathrm{d} \tilde{\rho}(x, s)}{\mathrm{d} x}+\frac{s+\Delta v_{x}}{\bar{v}(x)} \tilde{\rho}(x, s)=-\frac{\mathrm{d} \bar{\rho}(x)}{\mathrm{d} x} \frac{\tilde{v}(x, s)}{\bar{v}(x)}
$$

The solution of the homogenous part of Eq. (33) would be

$$
\tilde{\rho}(x, s)=\rho_{0}(s) \mathrm{e}^{-m x}
$$

with

$$
\begin{aligned}
& \rho_{0}(s)=\tilde{\rho}(0, s) \\
& m=\frac{s+\Delta v_{x}}{\bar{v}(x)}
\end{aligned}
$$

if $m$ (or $\bar{v}$ ) did not depend on $x$. Since the latter assumption is violated and Eq. (33) is not homogenous, the candidate for the solution is

$$
\tilde{\rho}(x, s)=\rho^{\prime}(x, s) \mathrm{e}^{-m x}
$$

Inserting Eq. (37) into Eq. (33), and taking into account

$$
\frac{\mathrm{d} m}{\mathrm{~d} x}=-\frac{s+\Delta v_{x}}{\bar{v}^{2}} \frac{\mathrm{d} \bar{v}}{\mathrm{~d} x}=-\frac{m}{\bar{v}} \frac{\mathrm{d} \bar{v}}{\mathrm{~d} x}
$$

yields

$$
\begin{aligned}
& \frac{\mathrm{d} \rho^{\prime}(x, s)}{\mathrm{d} x} \mathrm{e}^{-m x}+\rho^{\prime}(x, s) \mathrm{e}^{-m x}\left(-m-x \frac{\mathrm{d} m}{\mathrm{~d} x}\right)+m \rho^{\prime}(x, s) \mathrm{e}^{-m x}=-\frac{\mathrm{d} \rho(x)}{\mathrm{d} x} \frac{\tilde{v}(x, s)}{\bar{v}(x)} \\
& \frac{\mathrm{d} \rho^{\prime}(x, s)}{\mathrm{d} x}=-\rho^{\prime}(x, s) m \frac{x}{\bar{v}} \frac{\mathrm{d} \bar{v}}{\mathrm{~d} x}-\mathrm{e}^{m x} \frac{\mathrm{d} \bar{\rho}(x)}{\mathrm{d} x} \frac{\tilde{v}(x, s)}{\bar{v}(x)}
\end{aligned}
$$

Since $\mathrm{d} \bar{v} / \mathrm{d} x$ and $\mathrm{d} \bar{\rho} / \mathrm{d} x$ are very small, it follows from Eq. (40) that $\rho^{\prime}$ can be regarded as independent of $x$ (at least on the interval of interest $x \in\left[0, L_{\mathrm{p}}\right]$ ). Therefore, the solution (34) will be treated in the paper. 
Similarly, dependence of physical parameters of the pipeline $(L, R, C, T$, and $m)$ on $x$ shall be neglected in the rest of the paper since they are only functions of $\bar{v}$ and $\bar{\rho}$.

In the following, only the deviation model will be considered. To simplify the notation, the tildes will be omitted in the equations. The varaibles $p, v$, and $\rho$ will stand for the deviations of the respective variables from the stationary values.

The next step in the derivation of a simple model of the pipeline is the analytical solution of linear Eqs. (29) and (30). Performing the Laplace transformation on them yields

$$
\begin{aligned}
& (L s+R) V(x, s)=-\frac{\mathrm{d} P(x, s)}{\mathrm{d} x}-T \rho(x, s) \\
& C s \cdot P(x, s)=-\frac{\mathrm{d} V(x, s)}{\mathrm{d} x}
\end{aligned}
$$

where stationary initial conditions $\bar{v}$ and $\bar{p}$ were assumed, i.e. $v(x, 0)=0, p(x, 0)=0$. By differentiating Eqs. (41) and (42) with respect to $x$

$$
\begin{aligned}
& (L s+R) \frac{\mathrm{d} V(x, s)}{\mathrm{d} x}=-\frac{\mathrm{d}^{2} P(x, s)}{\mathrm{d} x^{2}}-T \frac{\mathrm{d} \rho(x, s)}{\mathrm{d} x} \\
& C s \frac{\mathrm{d} P(x, s)}{\mathrm{d} x}=-\frac{\mathrm{d}^{2} V(x, s)}{\mathrm{d} x^{2}}
\end{aligned}
$$

the following equations are obtained using Eqs. (41) and (42)

$$
\begin{aligned}
& (L s+R) \cdot C s \cdot P(x, s)=\frac{\mathrm{d}^{2} P(x, s)}{\mathrm{d} x^{2}}+T \frac{\mathrm{d} \rho(x, s)}{\mathrm{d} x} \\
& (L s+R) \cdot C s \cdot V(x, s)=\frac{\mathrm{d}^{2} V(x, s)}{\mathrm{d} x^{2}}-C s T \rho
\end{aligned}
$$

Taking into account Eq. (34) we obtain:

$$
\begin{aligned}
& (L s+R) \cdot C s \cdot P(x, s)=\frac{\mathrm{d}^{2} P(x, s)}{\mathrm{d} x^{2}}-T \rho_{0}(s) m \mathrm{e}^{-m x} \\
& (L s+R) \cdot C s \cdot V(x, s)=\frac{\mathrm{d}^{2} V(x, s)}{\mathrm{d} x^{2}}-C s T \rho_{0}(s) \mathrm{e}^{-m x}
\end{aligned}
$$

which are known as wave equations. Their solutions are

$$
\begin{aligned}
& P(x, s)=C_{1}(s) \mathrm{e}^{-n x}+C_{2}(s) \mathrm{e}^{n x}-\frac{T \rho_{0}(s) m}{n^{2}-m^{2}} \mathrm{e}^{-m x} \\
& V(x, s)=C_{3}(s) \mathrm{e}^{-n x}+C_{4}(s) \mathrm{e}^{n x}-\frac{C s T \rho_{0}(s)}{n^{2}-m^{2}} \mathrm{e}^{-m x}
\end{aligned}
$$

where

$$
n^{2}=(L s+R) \cdot C s
$$


The four expressions, $C_{1}(s), C_{2}(s), C_{3}(s)$, and $C_{4}(s)$ are not completely independent. By introducing (50) into (41) and by differentiating (49) with respect to $x$, we obtain

$$
\begin{aligned}
& (L s+R)\left[C_{3}(s) \mathrm{e}^{-n x}+C_{4}(s) \mathrm{e}^{n x}-\frac{C s T \rho_{0}(s)}{n^{2}-m^{2}} \mathrm{e}^{-m x}\right] \\
& =n C_{1}(s) \mathrm{e}^{-n x}-n C_{2}(s) \mathrm{e}^{n x}-\frac{T \rho_{0}(s) m^{2}}{n^{2}-m^{2}} \mathrm{e}^{-m x}-T \rho_{0}(s) \mathrm{e}^{-m x}
\end{aligned}
$$

From Eq. (52), the following relations between $C_{1}(s), C_{3}(s)$, and $C_{2}(s), C_{4}(s)$, respectively, are obtained

$$
\begin{aligned}
& \frac{C_{1}(s)}{C_{3}(s)}=\frac{L s+R}{n}=\sqrt{\frac{L s+R}{C s}}=Z_{K} \\
& \frac{C_{2}(s)}{C_{4}(s)}=-\frac{L s+R}{n}=-\sqrt{L s+R C s}=-Z_{K}
\end{aligned}
$$

since

$$
T \rho_{0}(s) \mathrm{e}^{-m x}\left[\frac{(L s+R) C s}{n^{2}-m^{2}}-1-\frac{m^{2}}{n^{2}-m^{2}}\right] \equiv 0
$$

The term $Z_{K}$ is called the characteristic impedance. The complete solution is obtained applying the boundary conditions. First, using the boundary conditions for $x=0$

$$
\begin{aligned}
& P(0, s)=P_{0}(s)=C_{1}(s)+C_{2}(s)-\frac{T \rho_{0}(s) m}{n^{2}-m^{2}}=Z_{K}\left(C_{3}(s)-C_{4}(s)\right)-\frac{T \rho_{0}(s) m}{n^{2}-m^{2}} \\
& V(0, s)=V_{0}(s)=C_{3}(s)+C_{4}(s)-\frac{C s T \rho_{0}(s)}{n^{2}-m^{2}}
\end{aligned}
$$

the coefficients $C_{3}(s)$ and $C_{4}(s)$ are obtained in the following form

$$
\begin{aligned}
& C_{3}(s)=\frac{1}{2} V_{0}(s)+\frac{1}{2 Z_{K}} P_{0}(s)+\frac{T \rho_{0}(s)}{2 Z_{K}} \frac{1}{n-m} \\
& C_{4}(s)=\frac{1}{2} V_{0}(s)-\frac{1}{2 Z_{K}} P_{0}(s)+\frac{T \rho_{0}(s)}{2 Z_{K}} \frac{1}{n+m}
\end{aligned}
$$

Next, the boundary conditions for $x=L_{\mathrm{p}}$ are used in Eqs. (49) and (50)

$$
\begin{aligned}
& P\left(L_{\mathrm{p}}, s\right)=P_{\mathrm{L}}(s)=Z_{K}\left[C_{3}(s) \mathrm{e}^{-n L_{\mathrm{p}}}-C_{4}(s) \mathrm{e}^{n L_{\mathrm{p}}}\right]-\frac{T \rho_{0}(s) m}{n^{2}-m^{2}} \mathrm{e}^{-m L_{\mathrm{p}}} \\
& V\left(L_{\mathrm{p}}, s\right)=V_{\mathrm{L}}(s)=C_{3}(s) \mathrm{e}^{-n L_{\mathrm{p}}}+C_{4}(s) \mathrm{e}^{n L_{\mathrm{p}}}-\frac{C s T \rho_{0}(s)}{n^{2}-m^{2}} \mathrm{e}^{-m L_{\mathrm{p}}}
\end{aligned}
$$

By introducing (58) and (59) into (60) and (61) the inverse chain representation of the pipeline is obtained

$$
\begin{aligned}
P_{\mathrm{L}}(s)= & -Z_{K} V_{0}(s) \sinh \left(n L_{\mathrm{p}}\right)+P_{0}(s) \cosh \left(n L_{\mathrm{p}}\right) \\
& +\frac{T m \rho_{0}(s)}{n^{2}-m^{2}}\left[\cosh \left(n L_{\mathrm{p}}\right)-\frac{n}{m} \sinh \left(n L_{\mathrm{p}}\right)-\mathrm{e}^{-m L_{\mathrm{p}}}\right]
\end{aligned}
$$




$$
\begin{aligned}
V_{\mathrm{L}}(s)= & V_{0}(s) \cosh \left(n L_{\mathrm{p}}\right)-\frac{1}{Z_{K}} P_{0}(s) \sinh \left(n L_{\mathrm{p}}\right) \\
& +\frac{T m \rho_{0}(s)}{Z_{k}\left(n^{2}-m^{2}\right)}\left[\frac{n}{m} \cosh \left(n L_{\mathrm{p}}\right)-\sinh \left(n L_{\mathrm{p}}\right)-\frac{n}{m} \mathrm{e}^{-m L_{\mathrm{p}}}\right]
\end{aligned}
$$

Expressions (62) and (63) can be simplified by the evaluation of $n / m$ using Eqs. (36), (51), (25)-(27):

$$
\frac{n}{m}=\frac{\sqrt{(L s+R) C s}}{\left(s+\Delta v_{x}\right) / \bar{v}}=\frac{\sqrt{(\bar{\rho} s+\bar{\rho} \lambda|\bar{v}| / D) 1 / a^{2} \bar{\rho} s}}{\left(s+\Delta v_{x}\right) / \bar{v}}=\frac{\bar{v}}{a} \sqrt{\frac{s^{2}+\frac{\lambda|\bar{v}|}{D} s}{\left(s+\Delta v_{x}\right)^{2}}}
$$

Note that $|n / m| \ll 1$ since the sound speed is much bigger than the fluid speed $(a \gg \bar{v})$. Consequently, the terms with $n / m$ in square brackets in Eqs. (62) and (63) can be neglected. By taking into account

$$
\frac{m}{n^{2}-m^{2}}=\frac{1}{m\left(\left(\frac{n}{m}\right)^{2}-1\right)} \approx-m^{-1}=-\frac{\bar{v}}{s+\Delta v_{x}}
$$

and

$$
\frac{n}{Z_{K}}=C s
$$

Eqs. (62) and (63) take the following form

$$
\begin{aligned}
& P_{\mathrm{L}}(s)=-Z_{K} V_{0}(s) \sinh \left(n L_{\mathrm{p}}\right)+P_{0}(s) \cosh \left(n L_{\mathrm{p}}\right)-\frac{T \bar{v} \rho_{0}(s)}{s+\Delta v_{x}}\left(\cosh \left(n L_{\mathrm{p}}\right)-\mathrm{e}^{-m L_{\mathrm{p}}}\right) \\
& V_{\mathrm{L}}(s)=V_{0}(s) \cosh \left(n L_{\mathrm{p}}\right)-\frac{1}{Z_{K}} P_{0}(s) \sinh \left(n L_{\mathrm{p}}\right)-\frac{T \bar{v} \rho_{0}(s)}{Z_{k}\left(s+\Delta v_{x}\right)}\left(-\sinh \left(n L_{\mathrm{p}}\right)\right)
\end{aligned}
$$

The constant $\Delta v_{x}$ is very small but positive (what can be seen from Fig. 1). Consequently, $1 /\left(s+\Delta v_{x}\right)$ is a stable transfer function. In the time scope of interest this transfer function is equivalent to an integrator, and will be replaced by one in the equations of the model. This is the reason why $\Delta v_{x}$ was not neglected in the early phase of the model derivation. Similarly, the term $\mathrm{e}^{-m L_{\mathrm{p}}}$ in Eq. (67) becomes

$$
\mathrm{e}^{-m L_{\mathrm{p}}}=\mathrm{e}^{-s+\Delta v_{x} \bar{v} L_{\mathrm{p}}} \approx \mathrm{e}^{-L_{\mathrm{p}} \bar{v} s}=\mathrm{e}^{-\tau s}
$$

and can be interpreted as a pure delay system ( $\tau$ is the time needed for the fluid to reach the outlet from the inlet of the pipeline - transport delay).

Apart from Eqs. (67) and (68), an additional equation is needed for the complete description of the system. This equation defines the density at the pipeline outlet and can be obtained by setting $x$ to $L_{\mathrm{p}}$ in Eq. (34):

$$
\rho\left(L_{\mathrm{p}}, s\right)=\rho_{\mathrm{L}}(s)=\rho_{0}(s) \mathrm{e}^{-m L_{\mathrm{p}}}=\rho_{0}(s) \mathrm{e}^{-\tau s}
$$

Finally, we arrive to the noncausal (the transfer function matrix goes to $\infty$ as $s \rightarrow \infty$ ) representation of the pipeline:

$$
\left[\begin{array}{c}
P_{\mathrm{L}}(s) \\
V_{\mathrm{L}}(s) \\
\rho_{\mathrm{L}}(s)
\end{array}\right]=\left[\begin{array}{ccc}
\cosh \left(n L_{\mathrm{p}}\right) & -Z_{K} \sinh \left(n L_{\mathrm{p}}\right) & -\frac{T v_{0}}{s}\left(\cosh \left(n L_{\mathrm{p}}\right)-\mathrm{e}^{-\tau s}\right) \\
-\frac{1}{Z_{K}} \sinh \left(n L_{\mathrm{p}}\right) & \cosh \left(n L_{\mathrm{p}}\right) & \frac{T v_{0}}{Z_{k} s} \sinh \left(n L_{\mathrm{p}}\right) \\
0 & 0 & \mathrm{e}^{-\tau s}
\end{array}\right]\left[\begin{array}{c}
P_{0}(s) \\
V_{0}(s) \\
\rho_{0}(s)
\end{array}\right]
$$


The linearised model of the pipeline (71) can be written in one of the following four forms which differ from each other with respect to the model inputs (independent quantities) and outputs (dependent quantities):

1. Hybrid representation: Inputs $V_{0}, P_{\mathrm{L}}, \rho_{0}$ and outputs $V_{\mathrm{L}}, P_{0}, \rho_{\mathrm{L}}$ :

$$
\left[\begin{array}{c}
P_{0} \\
V_{\mathrm{L}} \\
\rho_{\mathrm{L}}
\end{array}\right]=\left[\begin{array}{ccc}
\frac{1}{\cosh \left(n L_{\mathrm{p}}\right)} & Z_{K} \tanh \left(n L_{\mathrm{p}}\right) & \frac{T v_{0}}{s}\left(1-\frac{1}{\cosh \left(n L_{\mathrm{p}}\right)} \mathrm{e}^{-\tau s}\right) \\
-\frac{1}{Z_{K} \tanh \left(n L_{\mathrm{p}}\right)} & \frac{1}{\cosh \left(n L_{\mathrm{p}}\right)} & \frac{T v_{0}}{Z_{k} s} \tanh \left(n L_{\mathrm{p}}\right) \mathrm{e}^{-\tau s} \\
0 & 0 & \mathrm{e}^{-\tau s}
\end{array}\right]\left[\begin{array}{c}
P_{\mathrm{L}} \\
V_{0} \\
\rho_{0}
\end{array}\right]
$$

2. Hybrid representation: Inputs $V_{\mathrm{L}}, P_{0}, \rho_{0}$ and outputs $V_{0}, P_{\mathrm{L}}, \rho_{\mathrm{L}}$ :

$$
\left[\begin{array}{c}
P_{\mathrm{L}} \\
V_{0} \\
\rho_{\mathrm{L}}
\end{array}\right]=\left[\begin{array}{ccc}
\frac{1}{\cosh \left(n L_{\mathrm{p}}\right)} & -Z_{K} \tanh \left(n L_{\mathrm{p}}\right) & -\frac{T v_{0}}{s}\left(\frac{1}{\cosh \left(n L_{\mathrm{p}}\right)}-\mathrm{e}^{-\tau s}\right) \\
\frac{1}{Z_{K}} \tanh \left(n L_{\mathrm{p}}\right) & \frac{1}{\cosh \left(n L_{\mathrm{p}}\right)} & -\frac{T v_{0}}{Z_{K} s} \tanh \left(n L_{\mathrm{p}}\right) \\
0 & 0 & \mathrm{e}^{-\tau s}
\end{array}\right]\left[\begin{array}{c}
P_{0} \\
V_{\mathrm{L}} \\
\rho_{0}
\end{array}\right]
$$

3. Impedance representation: Inputs $V_{0}, V_{\mathrm{L}}, \rho_{0}$ and outputs $P_{0}, P_{\mathrm{L}} \rho_{\mathrm{L}}$ :

$$
\left[\begin{array}{c}
P_{0} \\
P_{\mathrm{L}} \\
\rho_{\mathrm{L}}
\end{array}\right]=\left[\begin{array}{ccc}
Z_{K} \operatorname{coth}\left(n L_{\mathrm{p}}\right) & -Z_{K} \frac{1}{\sinh \left(n L_{\mathrm{p}}\right)} & \frac{T v_{0}}{s} \\
Z_{K} \frac{1}{\sinh \left(n L_{\mathrm{p}}\right)} & -Z_{K} \operatorname{coth}\left(n L_{\mathrm{p}}\right) & \frac{T v_{0}}{s} \mathrm{e}^{-\tau s} \\
0 & 0 & \mathrm{e}^{-\tau s}
\end{array}\right]\left[\begin{array}{c}
V_{0} \\
V_{\mathrm{L}} \\
\rho_{0}
\end{array}\right]
$$

4. Admittance representation: Inputs $P_{0}, P_{\mathrm{L}}, \rho_{0}$ and outputs $V_{0}, V_{\mathrm{L}}, \rho_{\mathrm{L}}$ :

$$
\begin{aligned}
{\left[\begin{array}{c}
V_{0} \\
V_{\mathrm{L}} \\
\rho_{\mathrm{L}}
\end{array}\right]=} & {\left[\begin{array}{ccc}
\frac{1}{Z_{K}} \operatorname{coth}\left(n L_{\mathrm{p}}\right) & -\frac{1}{Z_{K}} \frac{1}{\sinh \left(n L_{\mathrm{p}}\right)} & -\frac{T v_{0}}{Z_{K} s}\left(\operatorname{coth}\left(n L_{\mathrm{p}}\right)-\frac{1}{\sinh \left(n L_{\mathrm{p}}\right)} \mathrm{e}^{-\tau s}\right) \\
\frac{1}{Z_{K} \frac{1}{\sinh \left(n L_{\mathrm{p}}\right)}} & -\frac{1}{Z_{K}} \operatorname{coth}\left(n L_{\mathrm{p}}\right) & -\frac{T v_{0}}{Z_{K} s}\left(\frac{1}{\sinh \left(n L_{\mathrm{p}}\right)}-\operatorname{coth}\left(n L_{\mathrm{p}}\right) \mathrm{e}^{-\tau s}\right) \\
0 & 0 & \mathrm{e}^{-\tau s}
\end{array}\right] } \\
& \times\left[\begin{array}{c}
P_{0} \\
P_{\mathrm{L}} \\
\rho_{0}
\end{array}\right]
\end{aligned}
$$

It should be noted that the forms 1-4 represent causal models, whilst Eq. (71) represent a noncausal model. Therefore, the latter cannot be realised by means of simulation. This completes the derivation of 
the transfer functions of the linearised pipeline. The resulting transfer functions are transcendent. In the next section, their approximations by rational transfer functions will be given.

\section{Simplified pipeline model with lumped parameters}

In this section, the rational transfer functions of the pipeline will be derived since such transfer functions are much easier to simulate, e.g. when designing real time transient model based leak monitoring systems. There are seven different transcendent functions $\left(\mathrm{e}^{-\tau s}\right.$ is the eighth one, but this one will be left as it is, since it can be simulated directly - at least approximately) in the four pipeline forms of the previous section: $1 / \cosh \left(n L_{\mathrm{p}}\right),\left(1 / Z_{K}\right) \tanh \left(n L_{\mathrm{p}}\right), Z_{K} \tanh \left(n L_{\mathrm{p}}\right), Z_{K} \operatorname{coth}\left(n L_{\mathrm{p}}\right), Z_{K}\left(1 / \sinh \left(n L_{\mathrm{p}}\right)\right),\left(1 / Z_{K}\right) \operatorname{coth}\left(n L_{\mathrm{p}}\right)$, and $\left(1 / Z_{K}\right)\left(1 / \sinh \left(n L_{\mathrm{p}}\right)\right)$. Only admittance representation transfer functions, used in the next section for verification of the model, will be analysed here (hybrid representation transfer functions can be found in [3]). By expanding the transcendent transfer functions into a Taylor series we get

$$
\frac{1}{Z_{K}} \operatorname{coth}\left(n L_{\mathrm{p}}\right)=\sqrt{\frac{C s}{L s+R}} \operatorname{coth}\left(L_{\mathrm{p}} \sqrt{(L s+R) C s}\right) \approx \frac{\left(\frac{1}{2} L_{\mathrm{p}}^{2} L C+\frac{1}{24} L_{\mathrm{p}}^{4} R^{2} C^{2}\right) s^{2}+\frac{1}{2} L_{\mathrm{p}}^{2} R C s+1}{\frac{1}{3} L_{\mathrm{p}}^{3} R L C s^{2}+\left(L_{\mathrm{p}} L+\frac{1}{6} L_{\mathrm{p}}^{3} R^{2} C\right) s+L_{\mathrm{p}} R}
$$

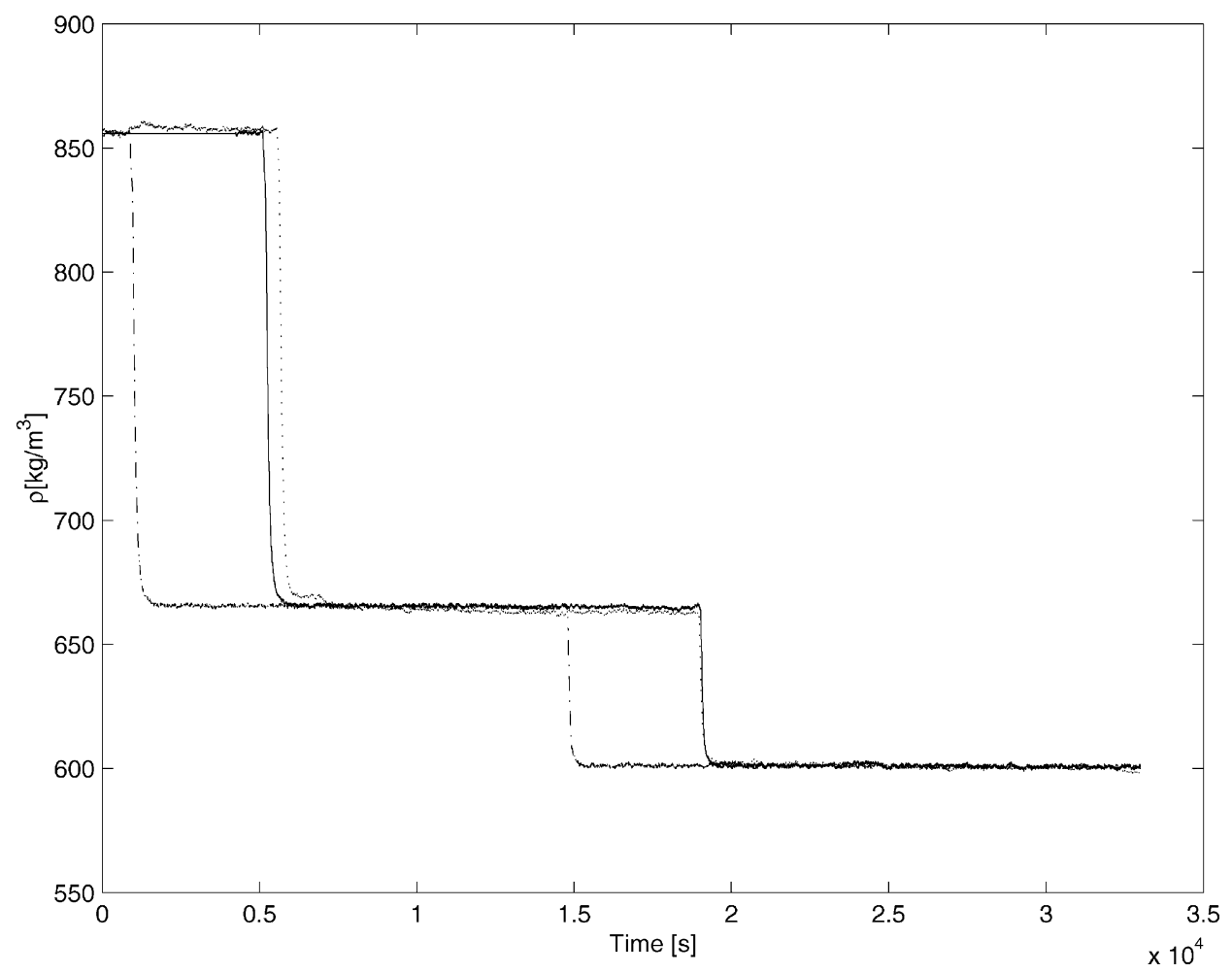

Fig. 2. The time courses of the density $\rho$ - measured $\rho$ at the inlet (dash-dot line), measured $\rho$ at the outlet (solid line) and simulated $\rho$ (dotted line). 
This transfer function describes the change of the fluid velocity at one end of the pipeline if the pressure is changing at the same end while the pressure at the other end and the density on the inlet remain constant.

$$
\frac{1}{Z_{K} \sinh \left(n L_{\mathrm{p}}\right)}=\sqrt{\frac{C s}{L s+R}} \frac{1}{\sinh \left(L_{\mathrm{p}} \sqrt{(L s+R) C s}\right)} \approx \frac{1}{\frac{1}{3} L_{\mathrm{p}}^{3} R L C s^{2}+\left(L_{\mathrm{p}} L+16 L_{\mathrm{p}}^{3} R^{2} C\right) s+L_{\mathrm{p}} R}
$$

This transfer function describes the change of the fluid velocity at one end of the pipeline if the pressure is changing at the other end while the pressure at the same end and the density on the inlet remain constant. It has a static gain $1 /\left(L_{\mathrm{p}} R\right)$ which corresponds to the static change of the velocity due to changing pressure.

\section{Validation of the simplified model}

The models were validated on a real pipeline with the following data: length of the pipeline $L_{\mathrm{p}}=$ $9854 \mathrm{~m}$, velocity of sound $a=1059 \mathrm{~m} / \mathrm{s}$, friction coefficient $\lambda=0.0158$, gravity constant $g=9.81 \mathrm{~m} / \mathrm{s}^{2}$, diameter $D=0.2065 \mathrm{~m}$, and inclination $\alpha=-0.00256 \mathrm{rad}$. Simulations of the plant were performed on

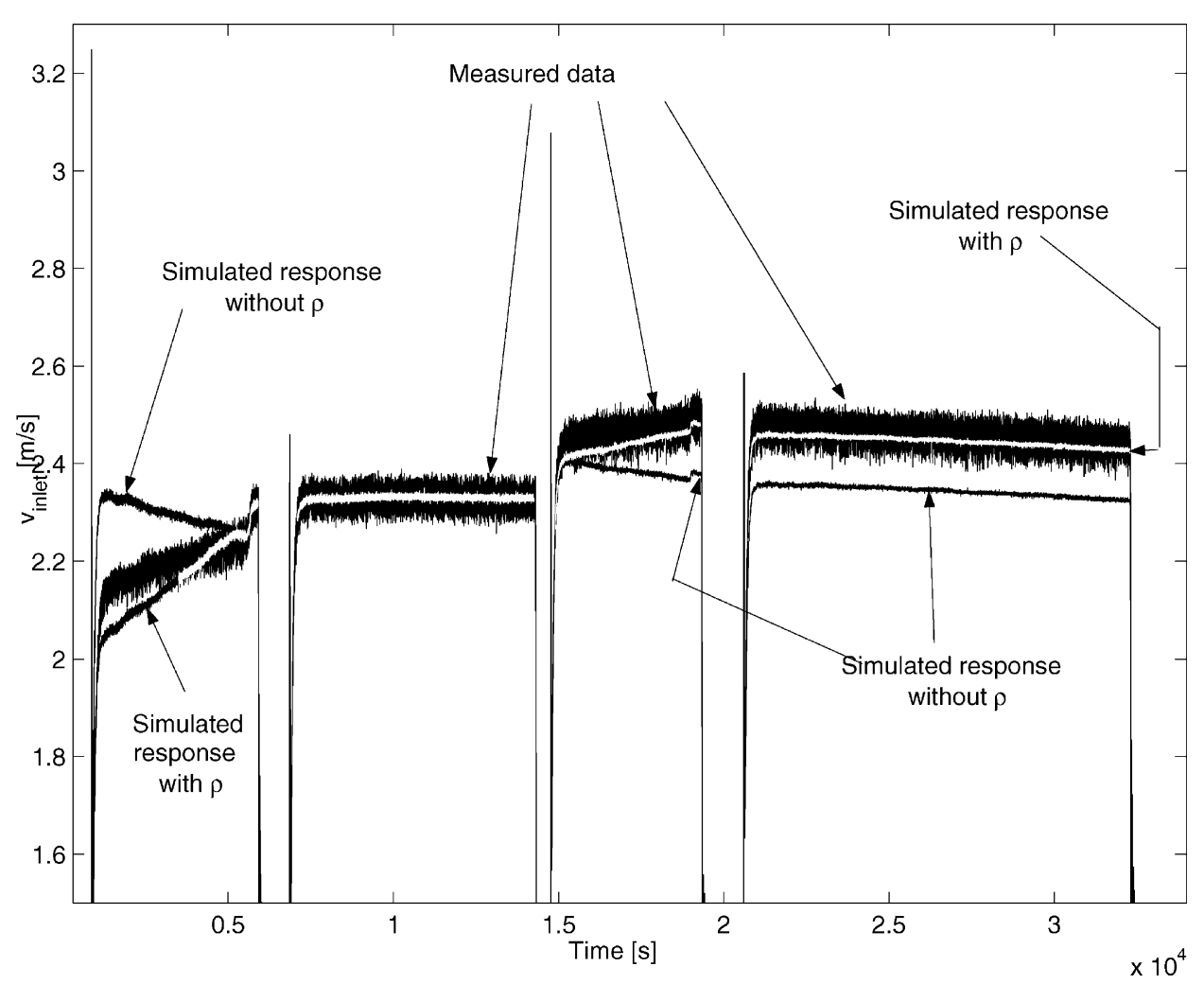

Fig. 3. The time courses of the fluid velocity at the inlet of the pipeline - measured velocity, model response without and with the consideration of the density $\rho$. 
the lumped parameter model in admittance form that was obtained by inserting Eqs. (76) and (77) into Eq. (75).

The most significant change in the fluid density $\rho$ occurs during a batch change, so the data was recorded in the operation phase where the batch was changed twice. Due to operational reasons the pipeline must be stopped before and after a batch change. The stationary operation between 7000 and 14,000 s was chosen as the operating point. Fig. 2 shows the time courses of the (measured) density $\rho$ at the inlet (dash-dot line) and outlet (measured density_-solid line; model response-dotted line) of the pipeline, respectively. The batch changes (1000-5300 and 14,800-19,000 s) can be seen clearly. The model response corresponds perfectly to the measured data for the second batch change, where the current operating conditions meet the chosen operating point. However, during the first change some miss-agreement can be noticed between the measured and the simulated density at the pipeline outlet. The difference is expectable since the system is not situated in the operating point where the linearised model was obtained.

Next, the velocity part of the lumped parameters model will be validated and compared to the model presented in [3]. The latter was obtained based on assumption that the density of the fluid being transported is constant all the time. The model proposed here takes the fluid changes into account. The comparison will allow us to estimate the benefit of the extended model. Simulation results of the both models will be compared to the real plant data.

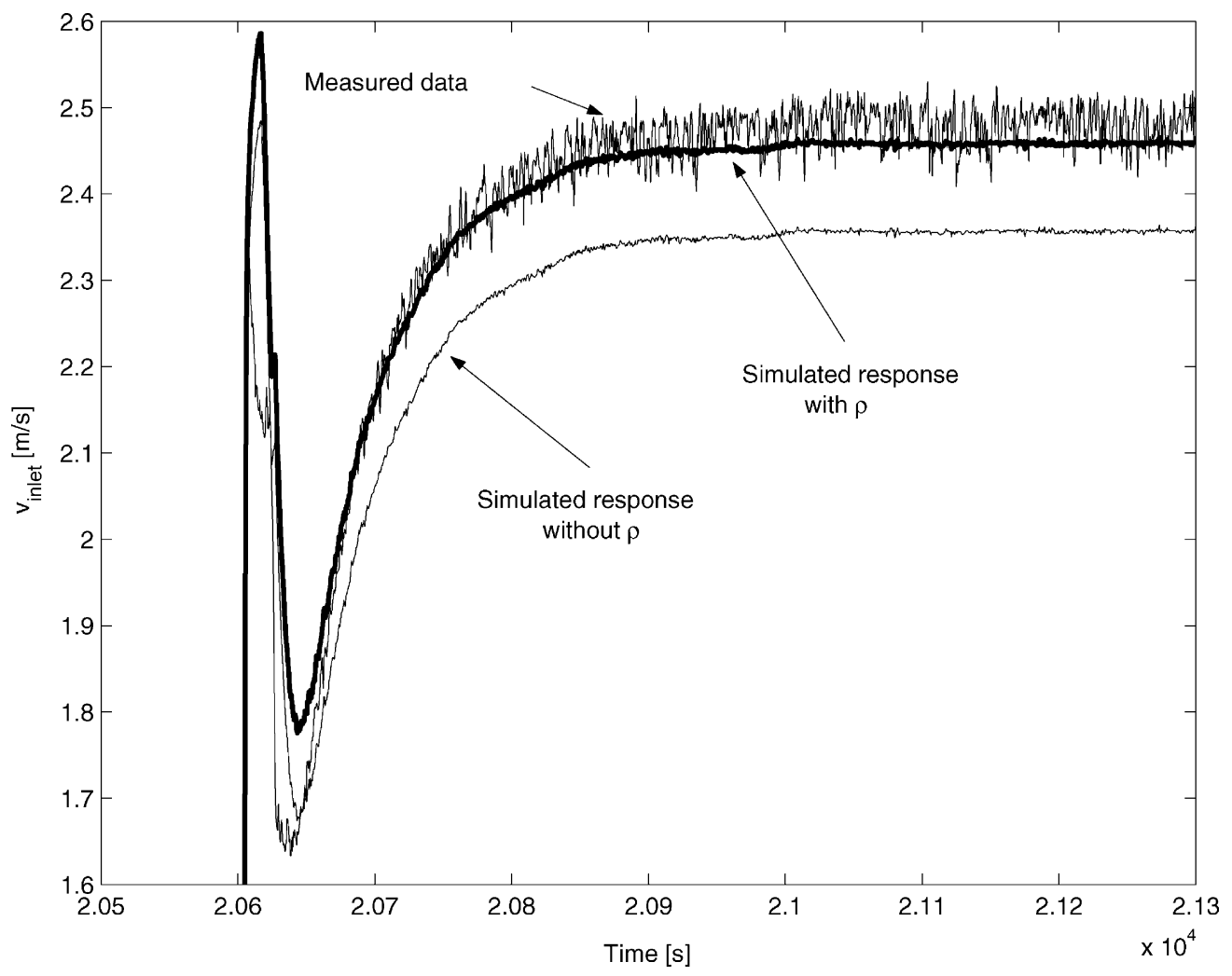

Fig. 4. A detail of Fig. 3. 
In Fig. 3 three time courses of the fluid velocity at the inlet of the pipeline are shown: the measured one and responses of two models (with and without the consideration of the density $\rho$ ). It can be seen that the proposed model which takes into consideration the density $\rho$ can cope with the changeable operating conditions whereas the model from [3] cannot. Note that the latter model performs very well between 7000 and 14,000 s where current density is approximately equal to the constant density of the simple model. But after the batch change the simulation results are not very satisfactory. In Fig. 4 a detail of the Fig. 3 is depicted, where the transient phase can be seen in detail. Good coincidence between the measured data and the proposed model response can be established.

\section{Conclusion}

The model of the multi-batch driven pipeline has been derived in the paper. To accommodate for the changes in the density, the latter is included in the model of the pipeline. The resulting model was the system of partial differential nonlinear equations. After linearisation and Laplace transformation a transfer function matrix was obtained that was transcendent. Simulations of such functions are quite complex and time consuming. This is why the lumped parameter approximation was found. The latter enables classical simulation and is therefore suitable for observer based leakage detection. It has been shown that the approximative model is capable of describing real plant dynamics. The experimentation has also shown that much better results are obtained by that model than the one proposed in [3] if different fluids are transported through the pipeline.

\section{References}

[1] V.L. Streeter, E.B. Wylie, Fluid Transients, McGraw-Hill, New York, 1978.

[2] A.H. Shapiro, The Dynamics and Thermodynamics of Compressible Fluid Flow, vol. 1, The Ronald Press Company, 1953.

[3] D. Matko, G. Geiger, W. Gregoritza, Pipeline simulation techniques, Mathematics and Computers in Simulation 52 (2000) 211-230.

[4] J.D. Anderson, Basic philosophy of CFD, in: J.F. Wendt (Ed.), Computational Fluid Dynamics, second ed., Springer-Verlag, Berlin, Heidelberg, 1996.

[5] V.L. Streeter, E.B. Wylie, Fluid Mechanics, eighth ed., McGraw-Hill, New York, 1985.

[6] D.H. Himmelblau, K.B. Bischoff, Process Analysis and Simulation, Deterministic Systems, Wiley, New York, 1968. 